\title{
Doxycycline or Ofloxacin for Outpatient Chlamydial Pelvic Inflammatory Disease? A Cost-Benefit and Cost-Effectiveness Analysis
}

\author{
Michael J. Rosenberg and Michael S. Waugh \\ Health Decisions, Inc. (M.J.R., M.S.W.), and Departments of Epidemiology and \\ Obstetrics-Gynecology, University of North Carolina (M.J.R.), Chapel Hill, NC
}

\begin{abstract}
Objective: The current Centers for Disease Control and Prevention (CDC) guidelines include 2 drugs, doxycycline and ofloxacin, for treatment of the chlamydial component of outpatient pelvic inflammatory disease (PID). Although ofloxacin costs about $\$ 90$ more than doxycycline, doxycycline is frequently associated with side effects and patient compliance with this drug is probably poor. Because clinicians have little information by which to judge the tradeoffs between price and compliance for these 2 antibiotics, we examined the impact of patient compliance in the evaluation of the costs and benefits of using each drug.

Methods: The incidence and direct costs of PID sequelae (infertility, ectopic pregnancy, and chronic pelvic pain) resulting after partially treated chlamydial PID were taken from previous estimates. For differing levels of antibiotic compliance, the probability of cure, probability of the occurrence of sequelae, and the associated cost of each were calculated. Because the relationship between partial antibiotic compliance and PID cure is unknown, we included 3 plausible relationships in our analyses. The sensitivity analysis was performed by varying key assumptions and examining the effect of each on future costs.

Results: The average probability of future PID sequelae attributable to chlamydia is slightly less than $2 \%$, with an associated cost of $\$ 1,272$. With an average compliance for doxycycline of $50 \%$, an improvement in compliance of as little as 1.8-3.5 percentage points (51.8-53.5\%), depending on the assumption used regarding partial compliance and cure, would make the use of ofloxacin less costly than doxycycline in the long run. Even with a cost difference of $\$ 90$ between the 2 drugs, a 10-percentage-point increase in compliance (to $60 \%$ compliance) with the more expensive drug would save $\$ 2.63$ for each $\$ 1.00$ spent.

Conclusions: Since the long-term costs of PID are likely to overshadow the immediate cost of providing treatment, physicians should carefully consider the likelihood of patient compliance in selecting an antibiotic. ๑ 1995 Wiley-Liss, Inc.
\end{abstract}

KEY WORDS

Antibiotic compliance, infertility, ectopic pregnancy, chronic pelvic pain

$P^{\mathrm{c}}$ elvic inflammatory disease (PID) is a serious reproductive health problem in the United States. Each year, an estimated 1.28 million women are treated as outpatients for episodes of acute PID. ${ }^{1}$

Direct and indirect costs, the latter including lost wages and lost value of household management, are estimated at $\$ 1.3$ billion annually for these cases. ${ }^{1}$ In addition to these costs, PID produces sequelae

Address correspondence/reprint requests to Dr. Michael J. Rosenberg, Health Decisions, Inc., 100 Europa Drive, Suite 525, Chapel Hill, NC 27515.

Portions of this work were presented at the Interscience Conference on Antimicrobials and Chemotherapy, Orlando, FL, October 5, 1994. 
that are often delayed, in the form of chronic pelvic pain, infertility, and ectopic pregnancy; these components add an additional annual cost of $\$ 3.8$ billion. ${ }^{1}$

Chlamydia trachomatis is an important contributor to PID. In the United States, it has been identified in $38 \%$ of hospitalized cases ${ }^{2}$ and in as many as $52 \%$ of women treated as outpatients. ${ }^{3,4}$ Infection with $C$. trachomatis is perhaps more insidious than with other forms of PID because of its less symptomatic nature. For example, Svennson et al. ${ }^{5}$ found that patients with chlamydial salpingitis often presented after a longer period (7-9 days) of abdominal pain. These patients less often had fever, but more often had elevated sedimentation rates (often to $30-50 \mathrm{~mm} / \mathrm{h}$ ). Yet, while the clinical findings were not impressive in patients with chlamydial salpingitis, laparoscopy revealed more pronounced inflammation of the fallopian tubes than expected from the clinical picture. Similarly, a higher proportion of women with chlamydial cervical infection had more demonstrable tubal infection than women with gonorrheal cervical infection. ${ }^{6}$

Because infection can rapidly cause irreversible tubal damage, antibiotics must be immediately started when PID is suspected. Many physicians intentionally overtreat, recognizing the difficulty of identifying an underlying salpingitis or endometritis based on physical and laboratory findings. ${ }^{7}$ Since few women diagnosed with PID, especially those treated as outpatients, undergo laparoscopy or other diagnostic or laboratory procedures that might help identify the microbiologic etiology, the treatment is by necessity empiric and broad enough to cover $C$. trachomatis as well as other pathogens linked etiologically with PID. Currently, the Centers for Disease Control and Prevention (CDC) recommends 2 regimens that include either doxycycline or ofloxacin for coverage of $C$. trachomatis. Both are highly effective against $C$. trachomatis, but ofloxacin costs approximately $\$ 90$ more than doxycycline. ${ }^{8}$

The effectiveness of either drug depends on how it is used. Patient compliance depends on a number of factors, including the occurrence of side effects, the degree of symptomaticity (perceived need), and the individual's degree of conscientiousness and health awareness. Even for medications that are regularly taken, compliance may be considerably less than optimal. For example, approximately one- fourth of patients using medications to prevent seizures, for whom the consequences of noncompliance are severe, did not comply with their prescribed regimen. ${ }^{9}$ Given the minimal degree of symptoms often associated with outpatient PID, particularly after the onset of symptom relief, the patient's perceived need to continue treatment may be reduced.

The few studies of drug compliance for the treatment of sexually transmitted diseases (STDs) support the notion that patient compliance is poor. One study of PID treatment in patients seen in an urban emergency department indicated an average compliance of only $50 \%$ with the doxycycline regimen (b.i.d. for 7 days). The occurrence of side effects was the strongest factor in compliance. ${ }^{10} \mathrm{~A}$ second study of erythromycin treatment for chlamydia and gonorrhea likewise indicated that the occurrence of side effects increased noncompliance. ${ }^{11}$

For acute infections, the occurrence of side effects may be a key determinant of compliance, and the limited evidence available suggests that doxycycline and ofloxacin differ in this regard. Two studies found that side effects were more prevalent with doxycycline regimens than with ofloxacin regimens. ${ }^{12,13}$ In these 2 comparative trials of oral ofloxacin ( $400 \mathrm{mg}$ b.i.d. for 10 days) or doxycycline (100 mg b.i.d. for 10 days), the side effects were approximately twice as common in the doxycycline group than in the ofloxacin group (respectively, $15 \%$ vs. $7 \%$ in one study and $26 \%$ vs. $16 \%$ in the other). Nausea and other gastrointestinal disturbances are commonly recognized as side effects of doxycycline and other tetracyclines. In the larger of the 2 studies, nausea and vomiting were significantly more common among patients using the doxycycline regimen. ${ }^{12}$

A clinician treating outpatient PID has little information on which to weigh the tradeoffs between patient compliance and cost. To address this issue, we present an analysis of the effect of patient compliance with doxycycline and ofloxacin on the future occurrence and cost of sequelae resulting from chlamydial PID.

\section{MATERIALS AND METHODS Costs of Sequelae}

The estimates of the costs of PID sequelae (chronic pelvic pain, ectopic pregnancy, and infertility) were taken from Washington and Katz. ${ }^{1}$ The direct costs 
per patient, reported in 1990, were inflated to 1995 dollars using the medical care component of the consumer price index by the formula " $1995 \$=$ $1990 \$ \times 1.081^{\mathrm{n}}$," where $n$ equals the elapsed number of years. These costs were then discounted to account for events occurring in the future by the formula "present value $=\left(1995 \$ \times 1.081^{\mathrm{n}}\right) /$ $(1.04)^{\mathrm{n}}$," where $n$ represents the estimated number of years in the future when the event will occur and $4 \%$ is the average rate of inflation for all items reported in the consumer price index. Chronic pelvic pain, including acute events such as salpingitis and tubo-ovarian abscess, is assumed to occur 3 years after the patient presents with PID; ectopic pregnancy is assumed to occur in 5 years; and infertility in 10 years. Thus, the present cost per case for each condition was $\$ 15,962$ for ectopic pregnancy, $\$ 2,010$ for infertility, and $\$ 14,754$ for chronic pelvic pain. Indirect costs, which refer to lost productivity and represent the value of output forgone by women with PID sequelae, were not considered in this analysis.

\section{Incidence of PID and Its Sequelae}

The estimates of the incidence of PID and PID sequelae were taken from the Hospital Discharge Survey conducted by the National Center for Health Statistics ${ }^{14}$ and from Washington and Katz. ${ }^{1}$ The latter rely on data published by the National Disease Therapeutic Index, which includes only initial visits to office-based physicians by women aged 15-44 years. Although the number of outpatient PID cases reported in 1993 by office-based physicians has declined by $7 \%$ since 1988 , this decline has been offset by an increase in the number of women relying on health maintenance organizations (HMOs), clinics, and emergency departments, leaving the overall incidence unchanged ( $T$. MacKay, CDC, personal communications).

The number of women hospitalized for PID in 1991 was used to estimate the total number of cases of chronic pelvic pain. ${ }^{14}$ The estimate of the annual incidence of PID-related ectopic pregnancy in 1991 was based on an assumption that $50 \%$ of the morbidity and mortality of ectopic pregnancy reported in the Hospital Discharge Survey is caused by PID. ${ }^{1}$ Along with the results of a study of women with laparoscopically verified PID who were followed for 10 years, we used Washington and Katz as our source in estimating that $20 \%$ of women with
PID will become infertile, with half (10\%) of these PID cases attributable to chlamydial PID. ${ }^{1,15}$

\section{Patient Compliance}

By average compliance, we mean the proportion of days, for all patients, that the medication was taken as prescribed. We found only one study of compliance with doxycycline for PID. This study, in an urban emergency department, found an average compliance of approximately $50 \% .{ }^{10}$ Complete compliance for the 7-day b.i.d. regimen was reported by $31 \%$ of the 386 patients surveyed, while $28 \%$ reported that they did not have their prescriptions for doxycycline filled. The remaining $41 \%$ reported that they stopped their medication early, after an average of 4.1 days. These side effects of doxycycline were the major determinants of noncompliance in patients who discontinued treatment early. No data were available regarding patient compliance with ofloxacin.

Because the relationship between partial antibiotic compliance and PID cure is unknown, we examined 3 plausible, but hypothetical, relationships (Fig. 1). For each relationship, we assumed that the probability of cure for chlamydia was equal for both doxycycline and ofloxacin at any given level of compliance. Full compliance was assumed to result in slightly less than full cure because, in some cases, even the early initiation of antibiotic therapy will be too late to prevent future problems, as reported in a number of studies. ${ }^{12,13,16}$ The clinical response in women with positive chlamydial cultures who presented with PID was estimated at $96 \%$, given full compliance. This estimate was based on an average of reported cure rates [18 of $18^{12}$ and 6 of $7^{13}$ patients cured with ofloxacin $(96 \%)$ and 15 of $17^{12}$ and 10 of $10^{13}$ cured with doxycycline (93\%)] when the regimens were taken as prescribed. Given the small number of patients and the absence of a significant difference in the clinical response, we used the higher average of reported cure rates.

\section{Change in Frequency and Cost of PID Sequelae With Compliance}

For a given level of compliance and a given relationship between partial compliance and cure (Fig. 1 ), the frequency and cost of chronic pelvic pain, ectopic pregnancy, and infertility were determined as follows. First, the probability of noncure was 


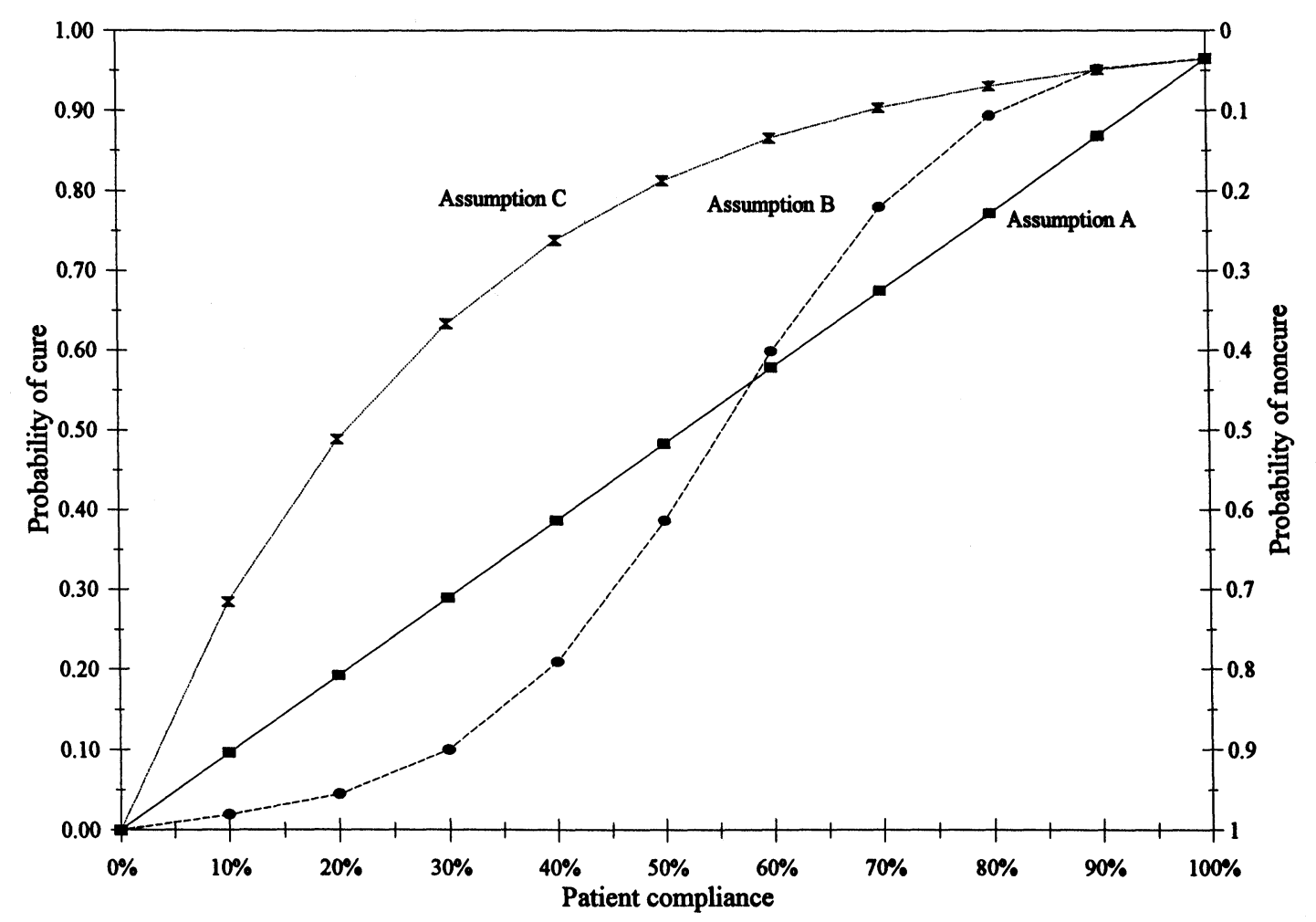

Fig. I. Three possible relationships between patient compliance and cure. Reduction of sequelae by increased drug compliance for each assumption calculated as discussed (see Materials and Methods).

determined from Figure 1. For example, under assumption $\mathrm{A}$, a $50 \%$ compliance is associated with a 0.52 probability of noncure. Second, the number of patients receiving a diagnosis of outpatient PID who were not cured was determined by multiplying the probability of noncure by the number of PID diagnoses each year $(0.52 \times 1.277$ million $\cong$ $660,400)$. Third, the probability of developing a given outcome was calculated by dividing the average probability of having that outcome, given a diagnosis of outpatient PID, by the probability of noncure. For ectopic pregnancy, this was 0.017 / $0.52=0.033(3.3 \%)$. Fourth, the number of cases of each outcome, such as ectopic pregnancy, was the product of the number of individuals who are not cured and the probability of developing an ectopic pregnancy among women who are not cured. For ectopic pregnancy, this was $660,400 \times 0.033$ 22,000 . Finally, the total cost of each outcome resulting from noncure due to inadequate compliance was the number of cases times the total direct cost per case. Again, for ectopic pregnancy, this cost was $22,000 \times \$ 15,962 \cong \$ 351$ million. The results of these calculations for each of the outcomes (ectopic pregnancy, infertility, and chronic pelvic pain), using one hypothetical relationship between compliance and the probability of cure (assumption A, Fig. 1), are summarized in Table 1. Identical calculations were performed for each hypothetical relationship between compliance and the probability of cure (assumptions B and C, Fig. 1, data not shown); the results are summarized in Figures 2 and 3 .

\section{RESULTS}

With 1.28 million women treated as outpatients each year for PID ${ }^{1,14}$ and an estimated $50 \%$ of these (half of all PID sequelae) primarily attributable to $C$. trachomatis, ${ }^{3,4}$ the probabilities of having an ectopic pregnancy, becoming infertile, or suffering chronic pelvic pain from chlamydia-associated PID, given a diagnosis of outpatient PID, were estimated at $1.7 \%, 10 \%$, and $5.4 \%$, respectively (Table 2). Using the total direct costs per case for each of the 3 PID sequelae, the average costs (total direct cost per case multiplied by the 
probability of developing the condition, given a diagnosis of PID for outpatient treatment) were $\$ 276, \$ 201$, and $\$ 795$, respectively; the total for all 3 diagnoses was $\$ 1,272$.

The probability of developing future complications is, however, affected by antibiotic compliance. Full compliance by all patients was presumed to cure nearly all chlamydial infections, resulting in an average cost of all 3 later complications of $\$ 86$. The small probability of later complications even with full compliance reflects the fact that, in some patients, damage will already have occurred even though treatment is promptly initiated. Zero compliance was presumed to cure none, with an average cost of $\$ 2,458$. Between these 2 extremes, different levels of compliance were associated with different numbers and costs of PID sequelae. Table 1, modeled for a single compliance-cure relationship, summarizes how the cost of each sequelae of inadequately treated PID depends on compliance. The differences in compliance from the $50 \%$ baseline substantially changed both the number of cases and the cost. For example, an increase in compliance from the $50 \%$ baseline to $60 \%$ for ectopic pregnancy would decrease the number of cases by 4,121 and the cost by $\$ 65.8$ million each year (Table 1 ).

Despite the greater initial expense of ofloxacin, treatment with this drug would be cost-beneficial if it could increase patient compliance by 3.5 percentage points or more over the $50 \%$ baseline compliance estimate for doxycycline (Fig. 2). Again, depending on which relationship between partial compliance and cure is analyzed, this increase might be as low as 1.8 percentage points for ofloxacin to be cost-beneficial. Using the most conservative of the 3 relationships tested (assumption A), an increase in average compliance from $50 \%$ to $60 \%(10$ percentage points) results in an average cost savings of $\$ 237$ which, offset by an increase in expenditures of $\$ 90$ per patient, yields a savings-to-cost ratio of almost $\$ 2.63$ for each $\$ 1.00$ spent. Likewise, an increase of 10 percentage points in compliance results in a reduction of $18.6 \%$ ( 41,000 cases) in the prevalence of sequelae (Fig. 3). Overall, this hypothetical increase of 10 percentage points in compliance could result in a savings of over $\$ 303$ million each year through a reduction in failed treatments and the prevention of PID sequelae, at an annual cost of almost $\$ 115$ million for the more expensive ofloxacin.

16 - INFECTIOUS DISEASES IN OBSTETRICS AND GYNECOLOGY

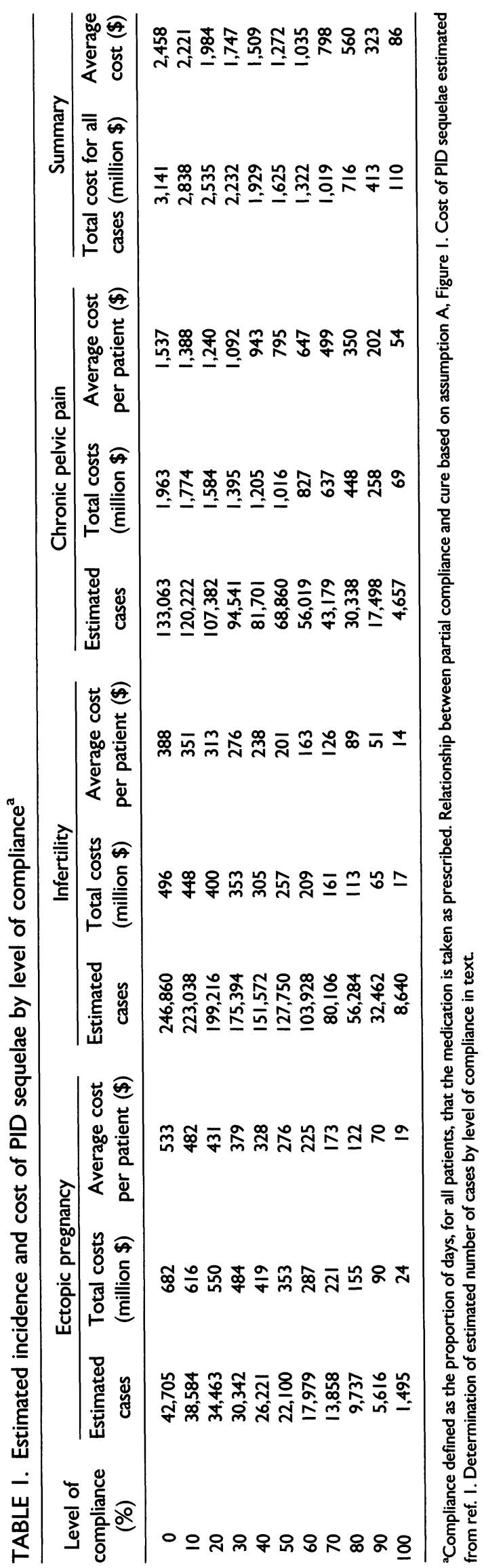




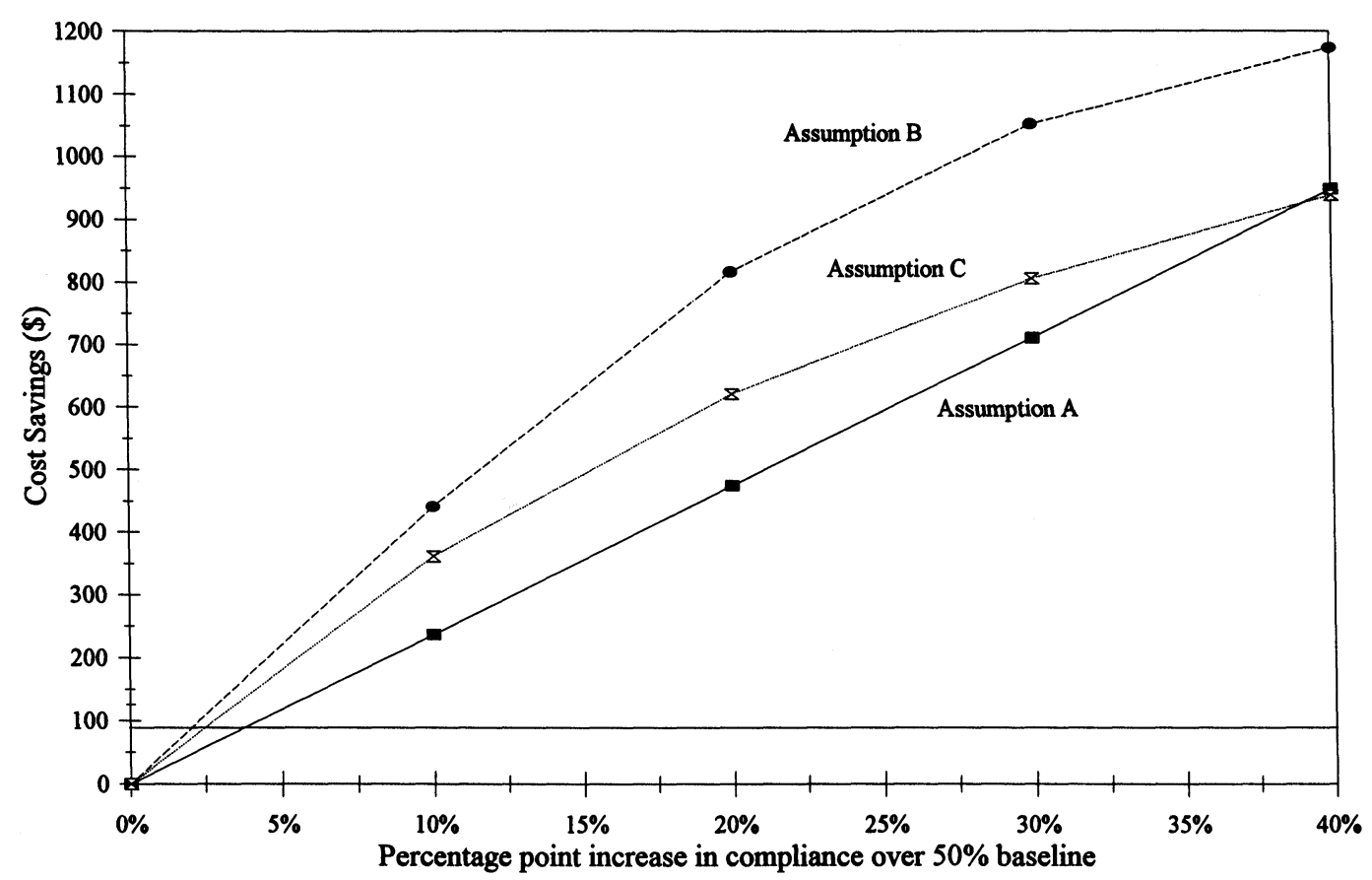

Fig. 2. Average savings per patient for PID sequelae by increased drug compliance. Reduction of sequelae by increased drug compliance for each assumption calculated as discussed (see Materials and Methods).

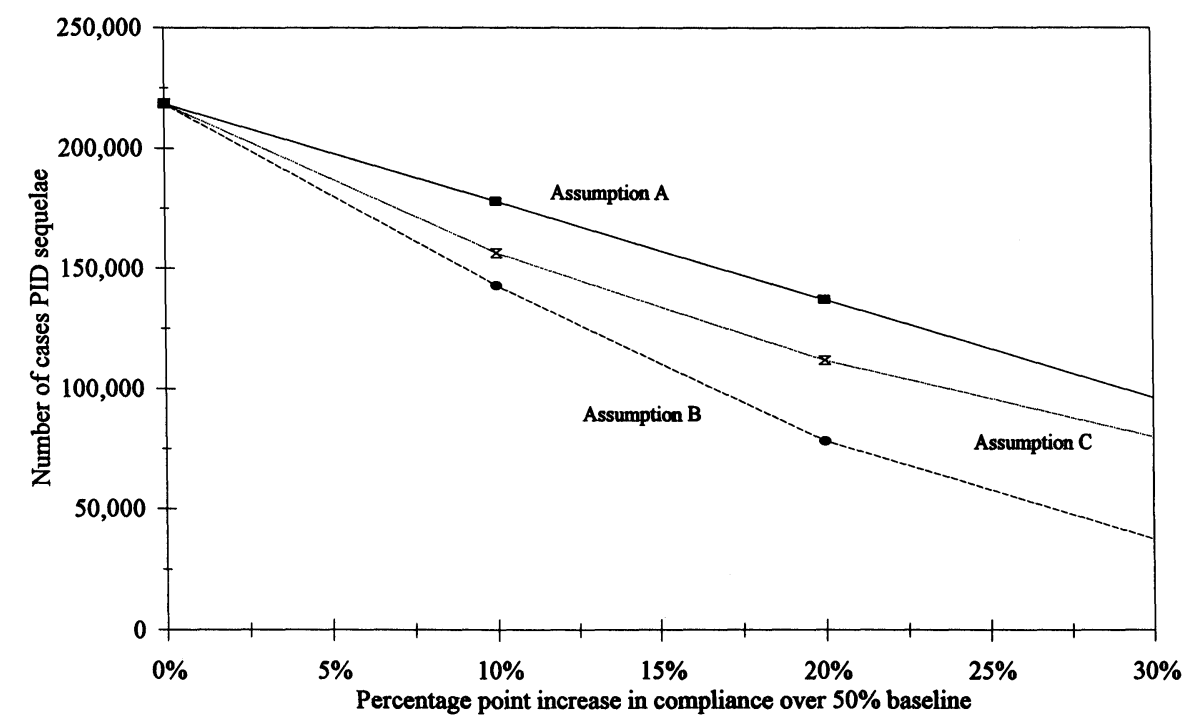

Fig. 3. Reduction in incidence of PID sequelae by increased drug compliance. Reduction of sequelae by increased drug compliance for each assumption calculated as discussed (see Materials and Methods).

\section{Sensitivity Analysis}

To determine how changing the key assumptions in the analysis might affect the conclusions, we examined a range of plausible values for each assumption
(Table 3). The 3 primary components of the analysis were baseline compliance with doxycycline, the frequency of PID sequelae, and the costs of these sequela. Changes of $\pm 20 \%$ in each of these compo- 
TABLE 2. Average frequency and cost of PID sequelae per patient, given outpatient diagnosis of PID

\begin{tabular}{|c|c|c|c|c|}
\hline & $\begin{array}{l}\text { Ectopic } \\
\text { pregnancy }\end{array}$ & Infertility & $\begin{array}{l}\text { Chronic } \\
\text { pelvic pain }\end{array}$ & Total \\
\hline No. of cases per year ${ }^{a}$ & 44,200 & 255,500 & 137,720 & 437,420 \\
\hline No. of cases attributable to chlamydia ${ }^{b}$ & 22,100 & 127,750 & 68,860 & 218,710 \\
\hline $\begin{array}{l}\text { Probability of developing condition, } \\
\text { given outpatient diagnosis of PID }\end{array}$ & 0.017 & 0.100 & 0.054 & \\
\hline Cost per case ${ }^{c}$ & $\$ 15,962$ & $\$ 2,010$ & $\$ 14,754$ & \\
\hline $\begin{array}{l}\text { Average cost, given outpatient diagnosis } \\
\text { of PID }\end{array}$ & $\$ 276$ & 201 & 795 & $\$ 1,272$ \\
\hline
\end{tabular}

aSee refs. I and 14.

${ }^{b}$ Assuming $50 \%$ of all cases of PID are attributable to chlamydial infection. See refs. 3 and 4.

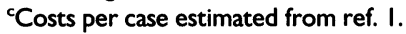

nents had minimal impact on the level of improved compliance necessary with ofloxacin to make its use cost-beneficial (the break-even point).

Changing the baseline compliance with doxycycline had no impact on our findings if the relationship between partial compliance and cure were linear (assumption A, Fig. 1). However, increasing the baseline compliance level by 20 percentage points increased the break-even point (assumptions B and C, Fig. 1), from $1.8 \%$ to $3.5 \%$ and from $2.3 \%$ to $4.5 \%$. Assuming the compliance with doxycycline to be 20 percentage points less than we assumed, we observed similar effects on the breakeven point. Varying other parameters, such as increasing or decreasing the costs and frequency of sequelae by 20 percentage points, similarly had minimal effects. Increases in the cost or frequency of sequelae reduced the level of compliance necessary to make the prescription of ofloxacin costbeneficial by 1 percentage point or less. Decreasing the cost or frequency of sequelae had an opposite, still negligible, effect.

\section{DISCUSSION}

Because inadequately treated PID due to $C$. trachomatis leads to expensive problems, even slight improvements in compliance would be associated with substantial reductions in the incidence and cost of later complications. In the choice between the 2 recommended drugs for treating the chlamydial component of PID, an improvement in compliance of as little as 1.8-3.5 percentage points over the $50 \%$ baseline (to $51.8-53.5 \%$ ) justifies the use of ofloxacin, which costs $\$ 90$ more than doxycycline. Even with such a cost discrepancy, an increase of as little as 10 percentage points in compliance with the more expensive drug would save $\$ 2.63$ for each $\$ 1.00$ spent. These estimates may be conservative because they do not include the indirect costs associated with PID sequelae. The inclusion of indirect costs would likely increase the cost savings associated with improved compliance. A sensitivity analysis confirmed these conclusions, regardless of how we varied the assumptions.

The strong financial impact of even slight improvements in patient compliance emphasizes the high costs of PID sequelae. Although the average probability of developing chronic pelvic pain, ectopic pregnancy, or infertility in a patient with outpatient PID is relatively low and not every patient who develops a problem will seek medical treatment, our calculations consider both these factors and emphasize the fact that each problem necessitates time-consuming, expensive, often complex procedures frequently over extended periods. In addition, the emotional toll for patients may be considerable. The cost of PID sequelae and the importance of adequate antibiotic treatment can be appreciated by the fact that the CDC recommends hospitalization for adolescents and other groups in whom compliance is traditionally poor. ${ }^{17}$

Our analysis is based on several assumptions. First, only minimal information is available about compliance with doxycycline and no comparative information is available for ofloxacin. Whether or not ofloxacin is associated with a better compliance rate than doxycycline does not alter the validity of the analysis, which emphasizes the importance and costliness of poor compliance. Nonetheless, the available studies of compliance in the treatment of PID consistently indicate that compliance is poor, with substantial proportions of patients failing to 
complete their medication and side effects increasing noncompliance. ${ }^{10,11}$

Second, the relationship between cure and varying levels of compliance is unknown. We considered 3 possibilities to address this uncertainty. For example, such a relationship may be linear (assumption A, Fig. 1) or "S"-shaped (assumption B, Fig. 1), with an initially low cure rate that increases rapidly over a short period and levels off after a certain threshold of compliance has been attained. Or, cure may be achieved rapidly with a relatively low level of compliance (the logistic curve, assumption C, Fig. 1). However, other relationships may exist. In addition, the precise relationship may vary according to individuals. Nevertheless, our finding that only minimal increases in compliance are necessary to markedly reduce the costs associated with PID sequelae, regardless of the relationships examined, suggests that patient compliancewhether an antibiotic is used as prescribedconsiderably overshadows the questions of the change in probability of cure at a specific period of time.

The baseline compliance rate of $50 \%$ compliance with doxycycline may be lower than that encountered in some groups. Although the study from which this figure was taken is the only one we were able to locate that directly addressed compliance for PID diagnoses, it involved patients at an urban emergency room. It is, however, similar to the $63 \%$ compliance rate noted for erythromycin in PID treatment from a study conducted in an STD clinic. In addition, both studies involved a 7-day regimen, so the compliance rates probably overestimate the level of compliance expected for the 14day regimen recommended by the CDC. Even if compliance differs from the baseline that we used, our sensitivity analysis confirmed our basic conclusions regarding the importance of compliance and savings attributable to a drug with improved compliance.

Compliance is obviated as an issue with azithromycin, which is effective against chlamydial cervicitis as an orally administered, single dose. However, insufficient clinical experience precludes our judging its efficacy in PID, as reflected by the fact that this drug is not yet included as one of the CDC's recommended treatments for PID.

The likelihood of a woman's coming to medical attention depends in part on her symptoms. Since 
infection with $C$. trachomatis is less likely than Neisseria gonorrhoeae to cause symptoms, the frequency of coinfection will affect whether a woman is treated. If women are not treated, the issue of compliance is moot. For an individual who receives treatment, however, the probability of compliance vs. the immediate cost of treatment and later costs of sequela due to inadequate treatment is important.

In this analysis, we approached costs from a broad perspective that included future as well as present costs. Although some settings, notably health departments, may focus on the immediate issue of antibiotic cost, such a view is narrow simply because others may bear the costs of future complications. From the broad perspective of a health care system, including comprehensive health providers such as HMOs, the argument for the use of antibiotics that decrease long-term costs is cogent. Other means of improving compliance need to be considered, including the physician's encouragement about the importance of the antibiotics, written materials to reinforce this information, and novel approaches to counseling, such as peer counselors for adolescents. ${ }^{18}$

With more information on compliance with a particular antibiotic regimen, the clinician may be able to tailor treatment to each individual patient, based in part on the patient's payment source for prescriptions. Office-based physicians must judge whether the patient, in the absence of insurance, federal support, or some other copayment plan, would be likely to have a prescription for ofloxacin or any other more expensive treatment filled. Clinicians working in managed care, publicly funded, or other capitation care environments should consider prescribing the alternative to doxycycline should it in fact prove to increase levels of compliance. By reducing the number of failed treatments and PID sequelae, the clinician also reduces the total costs that the health care system and potentially his or her individual institution may later absorb.

\section{ACKNOWLEDGMENTS}

We are grateful for the suggestions of Peter $\mathrm{Ma}$ zonson, M.D., and anonymous reviewers. This work was funded in part by a grant from OrthoMcNeil Pharmaceutical.

\section{REFERENCES}

1. Washington AE, Katz P: Cost of and payment source for pelvic inflammatory disease: Trends and projections, 1983 through 2000. JAMA 266:2565-2569, 1991.

2. Kiviat NB, Wolner-Hanssen P, Peterson M, et al.: Localization of Chlamydia trachomatis infection by direct immunofluorescence and culture. Am J Obstet Gynecol 154:865-873, 1986.

3. Bowie WR, Jones H: Acute PID in outpatients: Association with Chlamydia trachomatis and Neisseria gonorrhoeae. Ann Intern Med 95:685-688, 1981.

4. Moller BR, Mardh PA, Ahrons S, Nussler E: Infection with Chlamydia trachomatis, Mycoplasma hominis, and Neisseria gonorrhoeae in patients with acute pelvic inflammatory disease. Sex Transm Dis 8:198-202, 1981.

5. Svensson L, Westrom L, Ripa KT, Mardh PA: Differences in some clinical and laboratory parameters in acute salpingitis related to culture and serological findings. Am J Obstet Gynecol 138:1017-1021, 1980.

6. Mardh PA, Ripa KT, Svensson L, Westron L: Chlamydia trachomatis infection in patients with acute salpingitis. N Engl J Med 296:1377-1379, 1977.

7. Kahn JG, Walker CK, Washington AE, Landers DV, Sweet RL: Diagnosing pelvic inflammatory disease. JAMA 266:2594-2604, 1991.

8. Medical Economics Data, Inc.: Drug Topics Red Book 1993. Montvale, NJ: Medical Economics Data, Inc., 1993.

9. Cramer JA, Mattson RH, Prevey ML, Scheyer RD, Ouellette VL: How often is medication taken as prescribed? A novel assessment technique. JAMA 261:3273$3277,1989$.

10. Brookoff D, Kellerman A, Thorpe E: Compliance with doxycycline in outpatient treatment of pelvic inflammatory disease at an urban municipal emergency department (abstract). Sex Transm Dis 21:S123, 1994.

11. Katz BP, Zwickl BW, Caine VA, Jones RB: Compliance with antibiotic therapy for Chlamydia trachomatis and Neisseria gonorrhoeae. Sex Transm Dis 19:351-354, 1992.

12. Martens MG, Gordon S, Yarborough DR, Faro S, Binder D, Berkeley M: Multicenter randomized trial of ofloxacin versus cefoxitin and doxycycline in outpatient treatment of pelvic inflammatory disease. South Med J 86:604-610, 1993.

13. Wendel GD, Cox SM, Bawdon RE, Theriot SK, Heard $\mathrm{MC}$, Nobles BJ: A randomized trial of ofloxacin versus cefoxitin and doxycycline in the outpatient treatment of acute salpingitis. Am J Obstet Gynecol 164:1390-1396, 1991.

14. CDC: Pelvic inflammatory disease: Hospitalizations among women 15-44 years of age, United States, 19801991. National Hospital Discharge Survey. National Center for Health Statistics, CDC, Washington, DC.

15. Westrom L: Incidence, prevalence, and trends of acute pelvic inflammatory disease and its consequences in industrialized countries. Am J Obstet Gynecol 138:880892,1980 . 
16. Dodson M: Antibiotic regimens for treating acute pelvic inflammatory disease: An evaluation. J Reprod Med 39: 285-296, 1994.

17. Centers for Disease Control and Prevention. 1993 Sexually transmitted diseases treatment guidelines. MMWR 1993; 42(No. RR-14):75-83.
18. Jay MS, Durant RH, Shoffitt T, Linder CW, Litt IF: Effect of peer counselors on adolescent compliance in use of oral contraceptives. Pediatrics 73:126-131, 1984. 


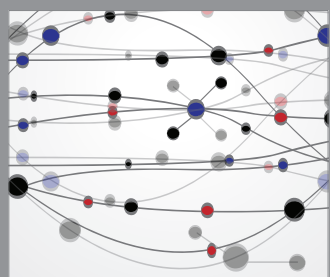

The Scientific World Journal
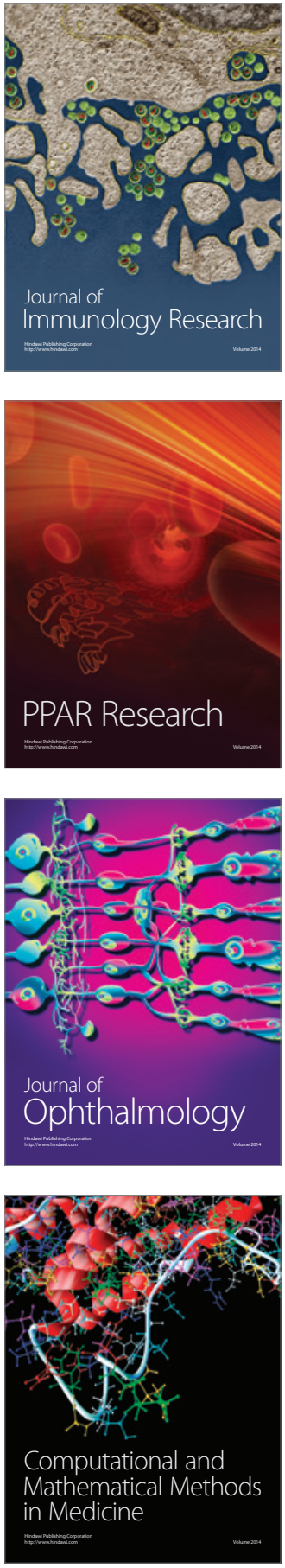

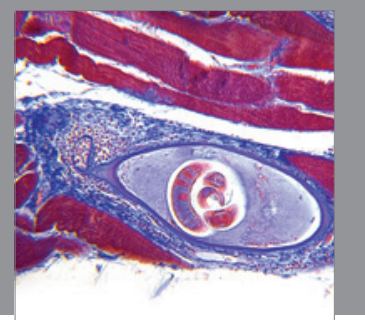

Gastroenterology

Research and Practice
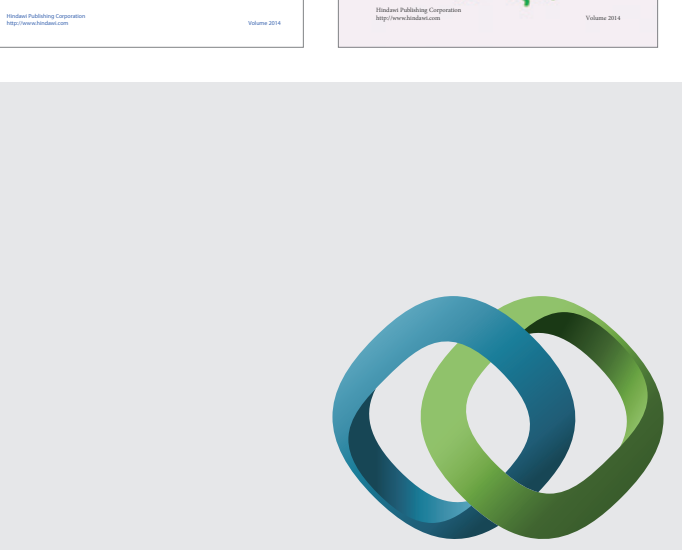

\section{Hindawi}

Submit your manuscripts at

http://www.hindawi.com
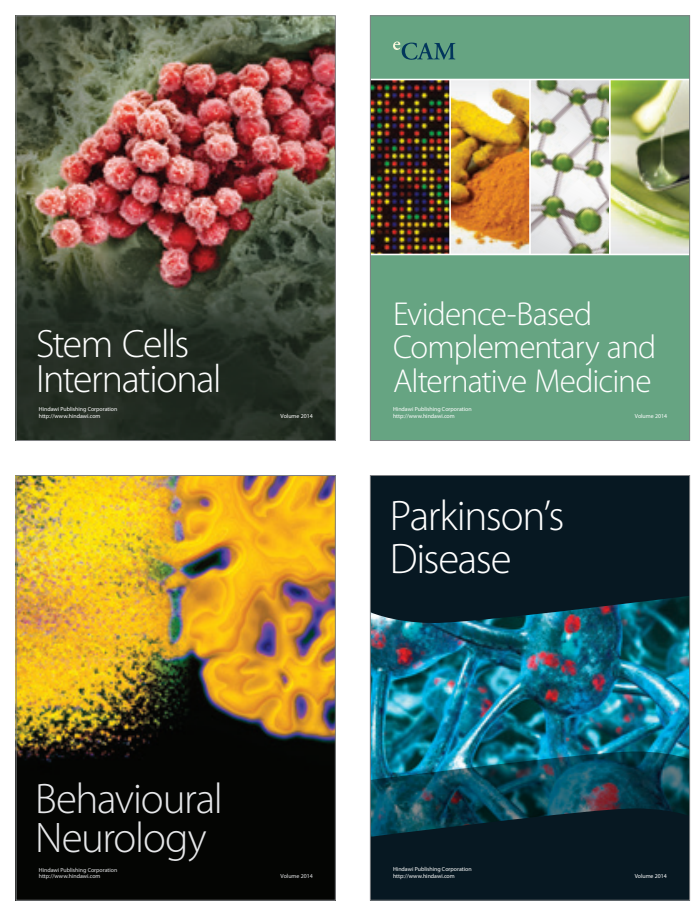

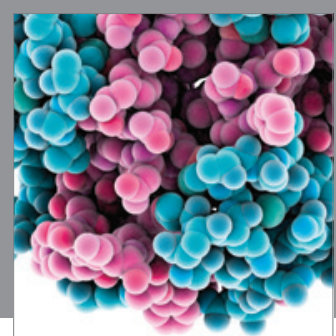

Journal of
Diabetes Research

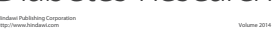

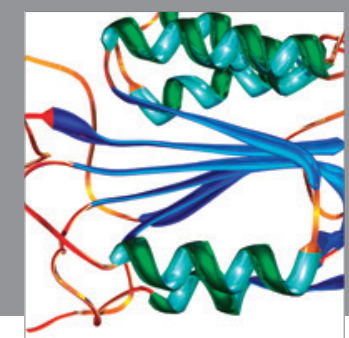

Disease Markers
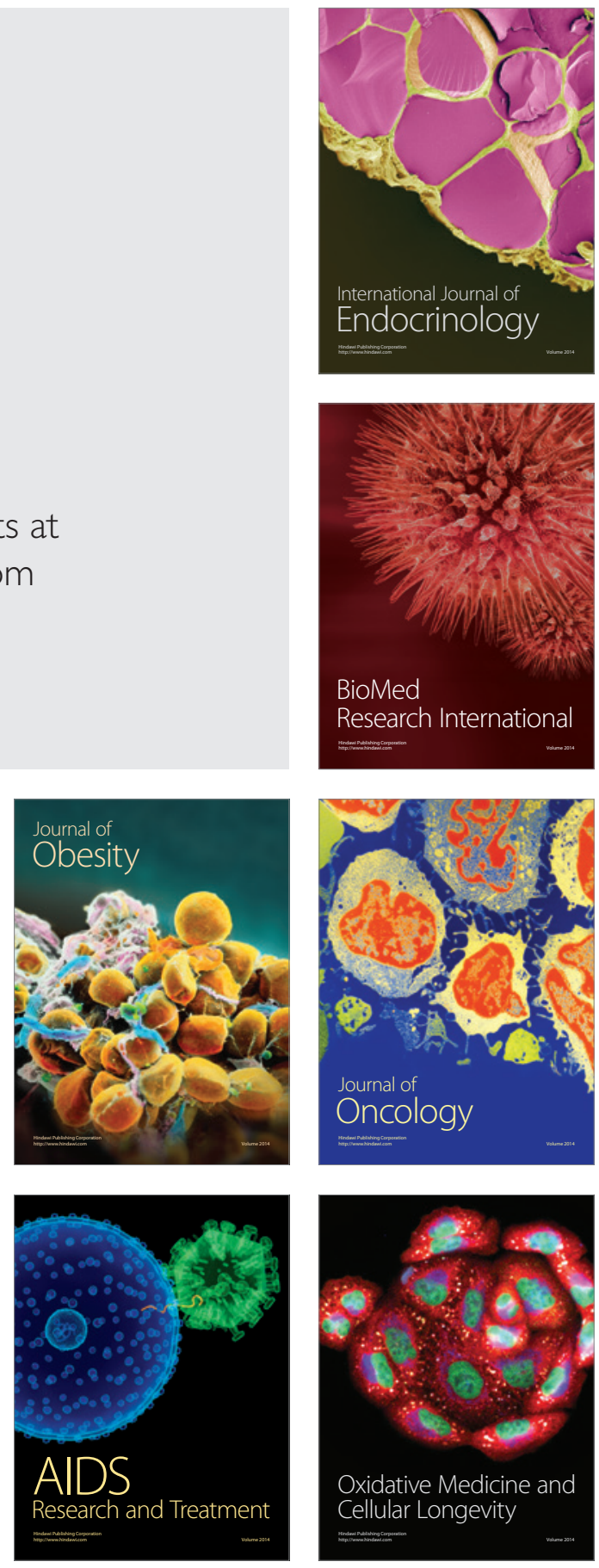